\title{
ULTRASONIC MONITORING OF LOCAL PHASE TRANSITIONS DURING VINYL CHLORIDE POOLYMERIZATION
}

\author{
P. Sladky
}

Department of Chemical Physics, Faculty of Mathematics and Physics, Charles University, Ke Karlovu 3, 12116 Praha 2, Crechoslovakia.

\begin{abstract}
Rēsumé. - Nous avons montré précêdemment que l'atténuation ultrasonore pouvait être mesurēe par une méthode d'impulsions ultrasonore dans une gamme de fréquences de quelques $\mathrm{MHz}$ pendant le processus de suspension polymërisation du chlorure de vinyle.

Dans cet article nous présentons les spectres en fonction du temps de la vitesse et de l'atténuation des ondes ultrasonores à la fréqeunce de $1 \mathrm{MHz}$ pour diverses conditions de polymërisations. Nous discutons qualitativement des modēles de centres de diffusion. Nous pensons que les pics les plus prononcés $\left(\alpha>60 \mathrm{~dB} \mathrm{~cm}^{-1}\right)$ sont liês à des transitions de phase locales liquide-vapeur du monomère dans le système sous polymérisation.
\end{abstract}

Abstracts. - It was shown earlier that ultrasonic absorption could be succesfully measured during the vinylchloride industrial /suspension/ polymerization by a pulse method in $\mathrm{MHz}$ frequncy range. In this report the ultrasonic absorption time-dependent spectra at $1 \mathrm{MHz}$ are presented for various polymerization conditions. Models of scattering centre are discussed quelitatively. It is believed that the most pronouned peaks $/ \alpha>60 \mathrm{~dB} / \mathrm{cm}$ are due to local liquid-vapour phase transitions of the monomer in the polymerizing system.

The suspension polymerization is an important technology of commercial poly (vinyl chloride) production. The vinyl chloride polymerization process is strongly exothermic and usually is performed in stainless steal reactors provided with thermostating mantel, back pipe cooler and stirring propeller. The physical properties of polymerization system are commonly describes in terms of pressure and temperature, as well as in terms of temperature of thermostating medium. Because of stirring action of the suspending agent, monomer droplets, dispersed in water phase are formed and maintained as isolated system during the polymerization until they become the final resin beads. Therefore, the local (nonequilibrium) thermodynamik properties of polymerization system such as are related to the morphology of the VC/PVC particles have an important bearing upon processability properties of the finished product and on the rate of removal of residual vinyl chloride. On the other hand, propagation of bulk longitudinal acoustic waves is mainly determined by the local properties of the system within the wavelength, thus providing the information about the dispersed phase. In our earlier paper /1/ we have shown that attenuation of acoustic waves in medium ultrasonic range can be successfully measured during vinyl chloride commercial suspension polymerization. In this report the ultrasonic time-dpendent spectra at $1 \mathrm{MHz}$ are presented and discussed for several polymerization conditions.
The measurements were carried out by a puise transmission technique using MATEC ultrasonic comparator electronics. Both attenuation and travel time of rf.ultrasonic pulses between transmitting and receiving transducers were measured by comparison methods and recorded manually from oscilloscope screen. Rf. pulses (of approx.5 sec duration and $500 \mathrm{~Hz}$ repetition rate) of longitudinal ultrasonic waves were generated and received by piezoceramic transducers of $800 \mathrm{kHz}$ fundamental frequen$c y$. The transducers were cemented to stainless steel buffer rods with plane-parallel ends. The ultrasonic apparatus was calibrated agints distilled water. The acoustic and electric insulation of transmitting and receiving transducers was better than $60 \mathrm{~dB}$ at $1 \mathrm{MHz}$. Relative error of attenuation meausurements was less than $2 \%$. U1trasonic pulse travel time was measured with approx. $1 \%$ accuracy. Typical courses of ultrasonic attenuation $\alpha(t)$ and travel time $\tau(t)$ spectra during the polymerization $A$ (porous PVC particles) are shown in fig. 1 The arrow on the time scale denotes the time, when the polymerization temperature was reached. The $\alpha(t, A)$ curve is characteristic though a large maximum appearing approx. one hour before the end of the polymerization with the peak value well above $200 \mathrm{dBcm}^{-1}$. The narrow peak at the beginning of the process $A$, appearing during the heating of polymerization system, is most probably due to scattering of ultrasonic waves on monomer bubbles 


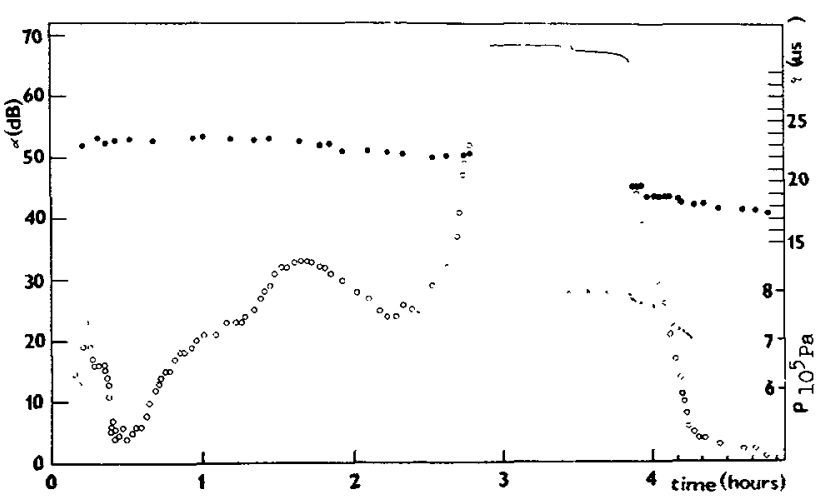

Fig. 1 U1trasonic attenuation /0000/ and trave time /.../ time dependent. spectra at $1 \mathrm{MHz}$ for vinyl chloride polymerization of type A./xxxx/ pressure in the reactor.

which are created transiently by eveporation of liquid monomer during the polymerization system heating. For other polymerization conditions the decay of this peak is observed at times which exceed the time when the polymerization temperature is reached (see for example Fig. 2). This fact indicates that boiling of the polymerization system at the beginning of the process is also accompainied by strong foaming of suspending agent in the primery stages of some polymerization technologies $/ 2 /$. The most strinking effect, observed during the polymerization $B$, is the presence of only small maximum at the end of the process. Since the polymerization technology $B$ produces more compact PVC particles, we can assume that the large (200min,A) maximum is again due to scattering of ultrasonic waves. However, in this stage of polymerization $A$, the scattering centers are formed in a consequence of the decrease of thermal exchange efficiency between the resin beads and suspending agent. Creation of both types of scattering centers $i . e$. resin beads filled with gaseous monomer as well as monomer bubbles is likely probable/3/. The above model of ultrasonic scattering centers which occur in the last stage of polymerization $A$ is supported by the observation of rapid increase of attenuation as a function of pressure decrease during degassing of final PVC product at the end of polymerization as it is illustrated in Fig. 3

Similar behaviour of uttrasonic attenuation is observed in freshly prepared suspensions of dry PVC particles during their degassing. Rather different attenuation vs. time curves were recorded during PVC polymerization under condition C (see fig 4)

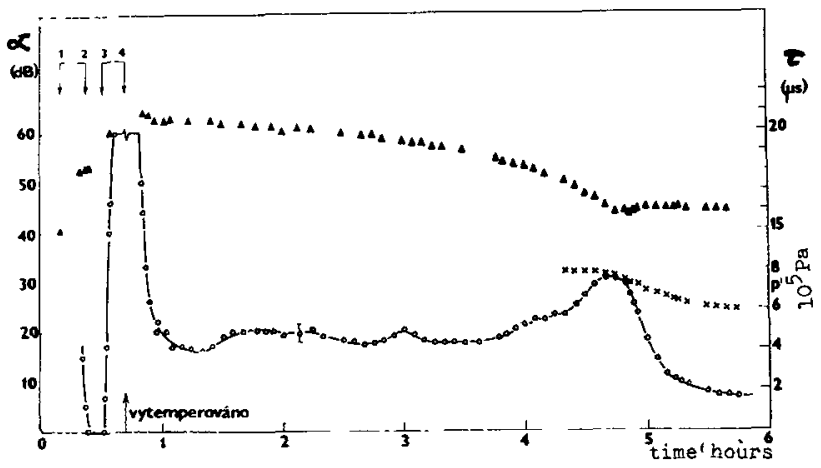

Fig. 2 Ultrasonic attenuation $/ 0000 /$ and travel time $/ \ldots . . /$ time dependent spectra at $1 \mathrm{MHz}$ for vinyl chloride polymerization of type B. Time interval 1-2-filling of the reactor with monomer, time interval 3-4-heating of the polymerization system. $/ x x x x /-$ pressure in the reactor

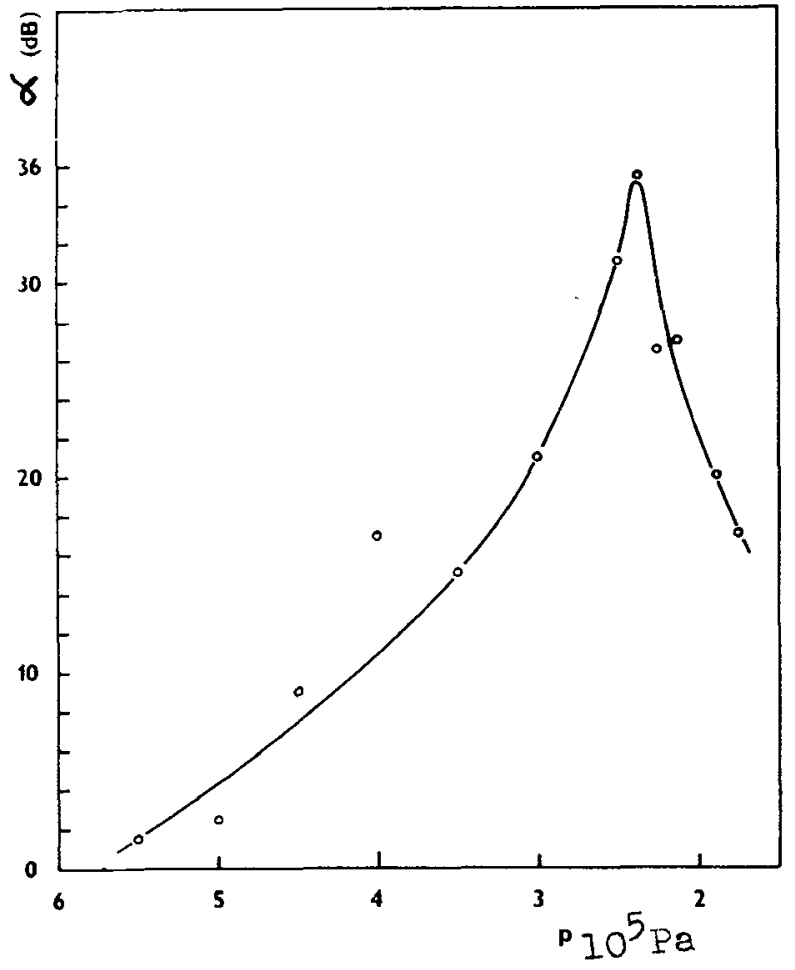

Fig. 3 U1trasonic attenuation vs. pressure dependence during slow degassing at the end of polymerization $A$.

In comparison with polymerization $A$ the $\alpha(t, C)$ curve indicates that the liberation of gaseous monomer begins almost at the end of the polymerization. For the detailed discussion of this effect, further experimental studies being conducted.

Although the present results bear preliminary character only, they have shown that the ultraso- 


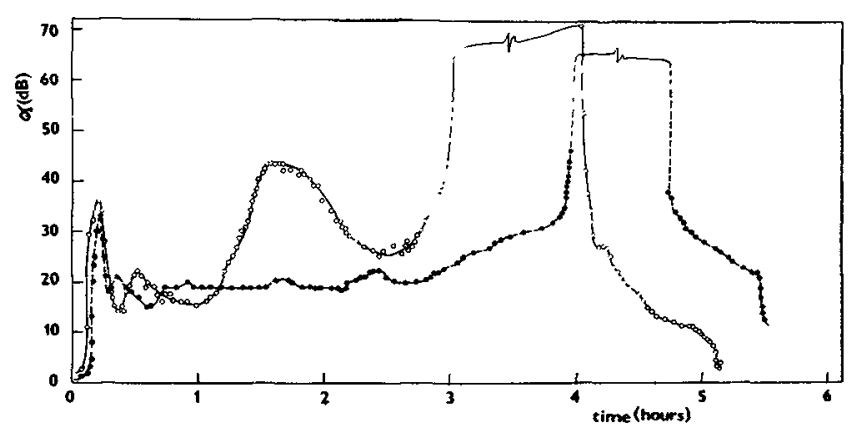

Fig. 4 Comparison of ultrasonic attenuation vs. time dependence for polymerization $\mathrm{A} / 0000 /$ and $c / \ldots .$.

nic measurements can provide many important informations about strongly nonlinear fluids such as are the vinyl chlorid polymerisation system under continuous stirring. It is also believed, that the present experimental results will stimulate more theoretical work on these problems because of their important applications.

\section{References}

/1/ Sladky P., Parma L., Pelant I., Zdrazil J., Docekal $\dot{j}$., "Ultrasonic Studies of Vinyl Chloride", Proceedings of the 5 th Conference of Czechoslovak Physicits, Kosice, August 1977

/2/ Sladky P., Pelant I., Parma L., Ultrasonics 16 (1979), 32

/3/ Zdrazil J., Stepanek J., S1adky P., "Participle Morphology and Acoustic Properties on Different Stages of Vinyl Chloride Suspension Polymerization". (to be published). 